Le Parlement a actuellement l'occasion de poser les jalons d'une Suisse en meilleure santé: les chambres fédérales examinent en effet pour la première fois en parallèle les législations sur l'alcool, sur les produits du tabac et sur les jeux d'argent. Les conséquences sur la santé de la nicotine, de la consommation excessive d'alcool et des pertes ruineuses d'argent au jeu sont connues. Le législateur est donc expressément invité à renforcer la prévention dans ces trois domaines, à permettre aux personnes concernées de trouver plus facilement de l'aide et à rendre plus difficile l'accès aux substances et pratiques à risque grâce à une politique cohérente des addictions.

\title{
Une chance unique pour une politique cohérente des addictions
}

\section{Barbara Weil}

Cheffe de la division Promotion de la santé et prévention

Les drogues et les addictions sont une réalité. Le souhait d'une société libérée de toute dépendance ne doit pas nous empêcher de nous pencher avec pragmatisme sur cet état de fait. Or à l'instar d'autres pays, la politique des addictions suscite de nombreuses

\section{L'addiction est une réalité qui concerne la société toute entière.}

controverses dans notre pays. En effet, les approches possibles sont aussi nombreuses que les valeurs et les croyances invoquées pour développer des solutions. En se concentrant sur des aspects émotionnels, moraux ou idéalistes, certains arguments ne tiennent cependant pas compte de la réalité ni de la situation souvent désespérée des personnes dépendantes.

Alors que les médecins sont confrontés quotidiennement au problème de la dépendance, le cadre légal et social a également un impact sur la prise en charge des personnes concernées. La dépendance est une maladie comme une autre: les personnes touchées ont besoin avant toute chose d'être aidées. De plus, il est toujours aussi important de replacer le problème des drogues illégales dans un contexte plus large, à savoir celui des substances psychoactives susceptibles d'induire une dépendance, comme l'alcool, le tabac ou encore les psychotropes. Sans oublier les nouvelles formes d'addiction "sans substance» comme l'addiction à internet ou aux jeux d'argent, en constante augmentation.

Dans le domaine de la prévention, les médecins ont un rôle central à jouer pour détecter les addictions avant qu'il ne soit trop tard. L'anamnèse systématique de la consommation permet d'intervenir avant que la dépendance ne s'installe et que les complications ne surviennent. Il est donc vivement conseillé d'aborder la question de manière franche et directe, en particulier avec les adolescents et les jeunes adultes confrontés à des situations de vie difficiles.
Les conditions-cadres légales revêtent également une grande importance pour prévenir les comportements à risque en instaurant des limites et des garde-fous afin d'empêcher certaines pratiques problématiques, dont l'accessibilité 24 h sur 24 des jeux d'argent sur internet, la multiplication des casinos (rappelons que la Suisse dispose de l'une des densités de casinos les plus élevées au monde) et la vente d'alcool à toute heure du jour ou de la nuit.

Depuis la publication du premier concept de la FMH sur la drogue en 1996, peu de choses ont réellement changé. La politique nous offre actuellement une nouvelle occasion de développer une approche crédible dans le domaine des addictions. En effet, les chambres fédérales examinent pour la première fois en parallèle les législations sur l'alcool, sur les produits du tabac et sur les jeux d'argent. Des interpellations ont été déposées à cet égard par plusieurs villes et cantons sur la question du cannabis.

Le marché influe sur le comportement des individus - notamment sur les plus vulnérables.

Mais aucune politique cohérente en matière d'addiction et de prévention n'aura de sens si les groupes d'intérêts s'évertuent à considérer ces trois lois comme si leur contenu n'avait aucun rapport, et s'il faut se battre pour chaque franc en faveur de la prévention et pour chaque mesure structurelle dont l'efficacité est pourtant déjà prouvée depuis longtemps.

En matière d'addiction, la FMH exige une action politique réaliste et globale, ainsi que des mesures de prévention coordonnées, et non contradictoires. L'addiction n'est pas uniquement un problème individuel mais bien un problème de notre société tout entière. 